\title{
Integrity of Supply Chain Visibility: Linking Information to the Physical World
}

\author{
Joris Hulstijn ${ }^{1}$, Sietse Overbeek ${ }^{1}$, Huib Aldewereld ${ }^{1}$, and Rob Christiaanse ${ }^{2}$ \\ 1 Faculty of Technology, Policy and Management, Delft University of Technology, \\ Jaffalaan 5, 2628 BX Delft, The Netherlands \\ \{J.Hulstijn, S.J.Overbeek, H.M.Aldewereld\}@tudelft.nl, \\ 2 VU University Amsterdam and EFCO Solutions, Singel 318, 1016 AE Amsterdam
}

r.christiaanse@efco-solutions.nl

\begin{abstract}
Regulatory compliance in international trade can be enhanced by facilitating electronic exchange of trade documents to increase the supply chain visibility. Crucial for acceptance of the supply chain visibility concept is trust in the reliability of the data. This depends on both the integrity of information (no data is altered illicitly) and integrity of the flow of goods (no goods are unknowingly added or taken away). The challenge is to determine how these concepts of integrity are interconnected. In this paper, we discuss control measures to ensure integrity of supply chain information and the related goods flow. Such controls consist of three components. First, only trusted traders take part. Second, technical control measures ensure internal consistency of the information system, i.e., the system maintains integrity constraints based on a model of the transactions taking place. Third, physical and organizational control measures mitigate the risk that events in the real world do not correspond to the reported transactions. The usefulness and adequacy of the approach is illustrated with two case studies: the use of electronic seals in the Smart-CM project, and the use of biometrics for authentication in the E-Link project at Schiphol Airport, the Netherlands.
\end{abstract}

Keywords: integrity, supply chain visibility, virtual data pipeline.

\section{Introduction}

Customs organizations are redefining their role regarding dealing with goods, not persons. On the one hand, they are supposed to facilitate international trade and reduce the related accounting burden for businesses. On the other hand, they must enhance safety and security of the international supply chain, in addition to their fiscal tasks. Generally, customs use a risk-based approach for selecting freights for inspection. These risk assessments require reliable data about transportation movements and the contents of containers. However, currently, the supply chain is organized in such a way that such data is not always reliable 13. For instance, the customs manifesto, which is the declaration that must be sent as an advance warning of importing goods is often incomplete or incorrect. 
One way to solve this problem is to enhance supply chain visibility: information systems help to trace evidence of the flow of goods along the supply chain [14]. When trading partners and regulators cooperate, it becomes possible to reuse commercially available information for regulatory purposes. This is called piggy backing 21. Commercially available information tends to be of better quality than information that is only demanded for regulatory reasons, because trading parties typically have countervailing interests. They will verify each others' data to see whether documents correspond to the actual flow of money and goods.

A particular approach to enhance supply chain visibility is the virtual data pipeline, developed in the CASSANDRA research project [14. The virtual data pipeline is essentially a data sharing architecture based on Linked Open Data [25. Linked Open Data refers to data published on the Web in such a way that it is machine readable, its meaning is explicitly defined, it is linked to other external datasets, and it can in turn be linked to from external datasets as well. Linked Open Data also refers to a set of best practices for publishing and connecting structured data. Here it will be used for exchanging trade documents among trusted traders. The regulator can view regulatory documents, e.g., customs declarations as well as selected commercial trade documents.

Figure 1 shows the virtual pipeline with the physical flow of goods underneath. The process follows the various stages of packing and sending the goods, freight forwarding, transportation, warehousing and distribution, until finally containers are unpacked by the consignee. The consigner is another word for seller of the goods and the consignee is the buyer.

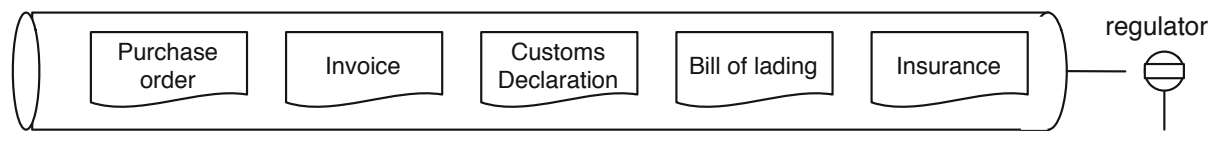

$?$

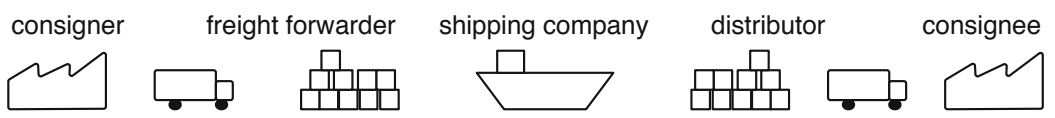

Fig. 1. Integrity of the virtual data pipeline concept

Crucial for acceptance of the data pipeline concept is the reliability of the data being exchanged. In this paper, we focus on the related notion of integrity. Literally integrity means 'undivided', 'whole' or 'without flaws'. The notion can be applied to the physical flow of goods. In that case it means that no goods may be added or taken from the flow, without this being noticed. Applied to data, integrity means: "Guarding against improper information modification or destruction, and includes ensuring information non-repudiation and authenticity" [8]. Information integrity involves accuracy (corresponds to reality) and completeness (covers all relevant aspects of reality), as well as timeliness (up-to-date) and validity (collected and processed according to regulations) 3. Therefore, 
establishing integrity requires an audit trail, a record of all steps in the process, which makes it possible to re-generate and verify information when needed.

In the context of supply chain visibility, the two notions of integrity should be interconnected, i.e., the information integrity and the integrity of the flow of goods. The central research question therefore is:

How can we make sure that the data being exchanged in the virtual data pipeline corresponds to all relevant aspects of the physical flow of goods?

The contribution of our approach is to define a business process model as a kind of intermediate stage. Steps in the process constitute a status update, which, when taken together form a well-defined economic transaction. Each status update is represented by a message or trade document, which is made available through the virtual data pipeline. Moreover, each status update is associated with an event or activity in the physical flow of goods of which evidence is also recorded. To undeniably link the different kinds of evidence, we need at least three kinds of control measures: (i) certification to ensure only known and trusted traders take part, (ii) control measures to ensure internal consistency, i.e., all data adheres to integrity constraints based on well-formed transactions [5], and (iii) control measures to ensure external consistency, i.e., to ensure that data will undeniably correspond to reality [5]. Our working hypothesis is that these three kinds of measures are necessary and sufficient, to establish integrity of the data about the flow of goods. A more general claim is that an intermediate conceptual model stated in terms of status updates, will in fact facilitate the design of control measures to ensure integrity of data about the flow of goods. The idea is that integrity can be established by aligning such status updates.

The approach is illustrated by two cases: (1) the E-Link project at Schiphol Airport, for redesigning customs procedures for air cargo export, and (2) the use of electronic seals for securing containers, as developed in the Smart-CM project. We also discuss challenges to the feasibility of the virtual data pipeline concept.

The remainder of the paper is structured as follows. In Section 2 we present our approach for ensuring integrity of the data about the flow of goods. Section 3 contains the two cases. Section 4 contains a discussion about feasibility. The paper ends with conclusions and recommendations for further research in section 5 .

\section{Linking Information Integrity and Physical Integrity}

The solution can be modeled in terms of a three layer architecture (Figure 2), compare Dietz [7]. On the bottom we have the physical layer with activities and events that constitute the transportation and distribution of goods. On the top we have the value layer with a business process which constitutes an economic transaction: generally a transfer of ownership or custody over the goods in return for money. The layer in the middle is the information layer. In this layer, evidence of events at the other two layers is being recorded and processed. 


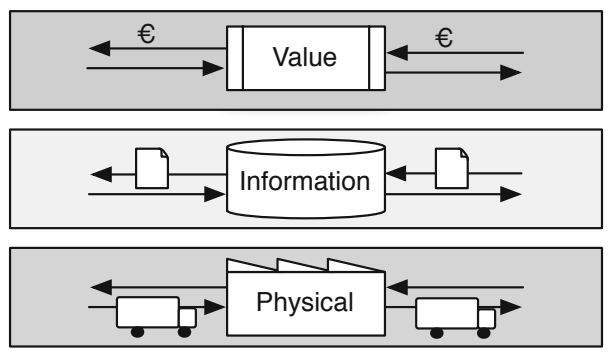

Fig. 2. Linking physical events through information exchange to value transactions

Generally, each relevant activity in the physical flow of goods is associated with a step in the business process. For example, when goods are unloaded from a truck, custody over the goods is transferred. Moreover, each status update is associated with a message or trade document, recorded in the participants' information systems. For example, when goods are unloaded the inventory master will sign a receipt for the goods, which is called a quittance. This piece of evidence will acquit the truck driver from the responsibility for the goods. Our purpose here is to reengineer the system of transfers and quittances in such way, that all major status changes in the business process are accounted for by undeniable evidence linked to physical events.

Each layer displays a kind of storage. On the physical layer a container, warehouse or ship contains inventory. In the middle we find a database. On the top, we find an abstract value repository, with financial accounts representing commitments and assets. At each layer, activities and events constitute status updates, which may add or take things away. Each storage concept can therefore be seen as a kind of data structure, subject to an invariant: a set of constraints which must be true at all times. Events may temporarily invalidate an invariant, but after a transaction the invariant will be restored. This is what constitutes a well-formed transaction: it restores the invariant, see Section 2.5 below.

For each storage concept, there are general continuity equations [22, compare reconciliation relations in accounting [20. For example, inventory at the end of the day should equal inventory at the beginning, with all incoming goods added and all outgoing goods subtracted. Or, the sum total of incoming goods of party $x$ should equal the outgoing goods of upstream partner $x-1$. Also for information similar equations can be formulated. For instance, the state of a database at the end of the day, should equal that of applying all updates to the begin state.

$$
\begin{array}{r}
e n d=\text { begin }+ \text { in }- \text { out } \\
\text { in }(x)=\operatorname{out}(x-1)
\end{array}
$$

The vertical relationship between layers is representational: events at the information layer represent status changes, both at the value layer above and the physical layer below. Conversely, the semantics of the concepts at the information layer can be expressed in terms of the value concepts or physical concepts. 
In other words: they provide evidence of status changes. The notion of evidence is based on so-called constitutive rules or counts-as rules [19]. Representations are social conventions, which only make sense within a certain social or institutional context. Therefore, it is important that the virtual data pipeline is a closed environment in which all participants are known.

\section{1 $\quad$ Trusted Traders}

The virtual data pipeline is essentially a closed system: participants are known and should in principle be trustworthy. In the context of the CASSANDRA project, traders are trusted when they are certified by the customs authority. They are so-called Authorized Economic Operators (AEO) [6]. In particular, the procedure uses a self-assessment which involves a risk analysis. After that, customs officials make an assessment whether the proposed control measures are sufficient given the risks on the basis of: (1) previous compliance behavior towards customs, (2) financial solvability, (3) reliable administration, and (4) adequate safety measures given the risks of the business.

In addition to certification by the authorities, we expect that commercial partners may also require a vetting procedure. After all, in general companies are reluctant to share information in a supply chain [16]. Participants may collaborate in one instance but be competitors in another instance. Note that a supply chain is flexible; the network infrastructure is stable. Therefore, the system should maintain who is collaborating with whom on a contract basis.

\subsection{Evidence and 'Counts as'}

In all complex social systems, events or situations in reality (brute facts) are given an additional social or institutional meaning (institutional facts). A quittance is an example. The brute fact is the piece of paper that one receives upon delivery of goods, while institutionally it is seen as a waiver of responsibility. This layering of institutional meaning upon real world events is based on John Searle's notion of 'constructing social reality' [19]. Social reality is created through counts-as statements: in institution $S$, fact or event $X$ counts as fact or event $Y$.

Table 1. Three notions of counts-as, producing evidence of a waiver of responsibility

\begin{tabular}{|l|l|l|}
\hline 1. & $\begin{array}{l}\text { "In institution } S \text {, a receipt created by procedure } P \\
\text { counts-as a quittance." }\end{array}$ & Constitutive \\
\hline 2. & "A quittance counts-as a waiver of responsibility." & Classificatory \\
\hline 3. & "In institution $S$ a receipt created by procedure $P$ & Proper \\
counts-as a waiver of responsibility." & Classification \\
\hline
\end{tabular}

The concept of counts-as has been thoroughly studied in formal logics [12, but also in computer science [1]. Grossi [12 first identified that the notion of countsas has three separate readings, see Table 1. The second premise is a general 
classification rule. It is universally applicable and could thus be formalized as ontological subsumption or logical implication, as in [1]. The conclusion, in 3, is a classification which only holds with respect to a given context. The first premise is not classificatory, but is 'constitutive': it can change institutional reality.

This formal link between brute facts and institutional facts is important for our topic, since, when auditing, one does not go looking for 'waivers of responsibility', but instead one tries to find evidence, such as 'receipts created by procedure $P^{\prime}$. In an electronic trading environment, messages have replaced the physical evidence of paper receipts, but their function remains. In other words, aligning events with economic status updates, is nothing but establishing constitutive rules, about what counts as evidence of a transaction.

\subsection{Technological and Physical Infrastructure}

The technological and physical infrastructure involves hardware like gates, fences, doors, locks and keys, ID passes etc. Such measures generally alter the environment in order to enforce some deserted behaviour. In general, organizational or procedural control measures like supervision make no sense, unless there is an environment containing necessary physical control measures. This holds in particular for security measures concerning the flow of goods. For instance, suppose that an radio frequency identification (RFID) reader should scan all boxes before they are cleared out of a warehouse to be exported. Installing such an RFID reader is useless when the goods are not guaranteed to pass through it. This can be achieved for instance by a system of conveyer belts. Similarly for people, a card reader for verifying ID cards makes no sense without additional gates and fences to make people pass through the gate. Physical security measures can be preventative (fence) and detective (camera systems; alarms). In both cases they help to maintain the integrity of the flow of goods, by ensuring that only authorized people may get access to the goods. The assumption is that authorized people will generally perform their duties; this assumption is warranted because all participants are certified AEO traders.

\subsection{Database Management}

At the data level, integrity is mostly maintained by features of the database management system (DBMS). Chen [4] distinguishes domain integrity, which requires that only syntactically and semantically correct data should be inserted in the database, and referential integrity, which implies that: "a piece of data cannot exist or be modified unless some precursor data value exists or some action is taken" 4]. A DBMS uses integrity constraints to verify the data and operations on the data [1]. These constraints are rules that define semantic properties to be satisfied by the data in the database in order to settle static integrity. Data is altered by updates, which together correspond to a database transaction. When regarding dynamic integrity, two things must be verified for any authorized database update. First, any newly entered input data must meet the applicable integrity constraints. Second, the transaction will guarantee that 
data will remain well-formed and integrity constraints are preserved [5]. Other traditional measures for maintaining data integrity are the mirroring technique, the checksum technique, the hamming codes method, and the hash function method 9]. These techniques are not deployed in a vacuum. There should be procedures to make sure these data integrity preserving techniques are not circumvented. In particular, users may only access and manipulate data through certified software applications [5]. This explains the high emphasis on change management procedures in modern information security.

\subsection{Business Process and Transactions}

Where do these integrity constraints come from? The answer is provided one level upwards, namely at the business process level. At the heart of every business lies the transaction. A transaction is a set of contracting relationships among individuals. It regulates an exchange of objects of value, for example money for goods. In order to perform a transaction, certain operational activities must take place. One could say that the transaction is being constituted by these activities. Conversely, using the terminology defined in Section 2.2. these operational activities count as a valid transaction. These operational activities also generate evidence which constitutes the audit trail. A transaction involves commitments by at least two parties. In all cases, some form of explicit confirmation is required to signal acceptance of a commitment. For example, an offer is responded to by a purchase order, which is subsequently accepted. After execution, again some confirmation is needed to signal acceptance of delivery [7/24]. For example, when signing for receipt of the goods (quittance), you indicate that the responsibility for the goods is transferred. Therefore confirmation evidence, like receipts or quittance, is crucial in establishing the economic status of the goods.

Business reality can be modeled as a value cycle: an interrelated system of flows of money and goods [220]. So-called accounts represent a state of a certain value to the company, such as inventory or accounts payable. An event is a change of state. For example, a sales event leads to an increase in accounts receivable and a decrease in inventory. Similarly, a purchase leads to an increase in inventory and an increase in accounts payable, etc. In addition, we have continuity equations for each of the states: begin $-e n d+i n-o u t=0$. A proper financial administration demands stable reconciliation relationships between these states or accounts. Because of the security aspect of the domain (customs inspections) we are especially interested in the completeness assertion: all goods being transported must be accounted for. Therefore, we believe that the Dutch tradition of owner-ordered accounting theory provides a good set of candidate reconciliation relations or continuity equations 22 . Unlike the U.S. manager-ordered accounting tradition, which mainly focuses on accuracy, owners are also interested in the completeness of stated revenues. In the owner-ordered tradition, there are various techniques of verifying completeness assertions by finding countervailing registrations to compare them against, see [2].

Recalculation and verification of the reconciliation relations on the basis of independent evidence is a strong detection measure to identify misstatements. 
That is essentially what an auditor or tax inspector does. But crucially, such verifications can also be built into the business processes and the software that supports it. This approach is called compliance by design [10.

\section{Illustrative Cases}

In the two cases that are presented we focus on the interrelationship between external validity and internal validity. Firstly, the integrity approach will be illustrated in a case concerning pre-announcements and biometric authentication and secondly it will be illustrated in a case where electronic seals are used.

\subsection{E-Link Project at Schiphol Airport}

Air cargo is a fast business. Every minute counts. Given the tight schedules of arriving and departing aircraft, delays or even uncertainties lead to losses. The E-Link project is trying to reduce paperwork and waiting times of air cargo handling at Schiphol Airport, using biometrics authentication and a system of preannouncement:1 The scenario makes use of the identity pass for truck drivers issued by Air Cargo Netherlands (ACN). Before joining ACN a cargo handler must be certified. For more information about the project we refer to [17. The E-Link scenario of air cargo delivery by truck to ground handlers at Schiphol Airport, runs as follows (Figure 33):

Step 1. The company sending the goods settles the commercial transactions and prepares the necessary freight documentation (air way bill, etc.).

Step 2. The company pre-announces the visit, entering all required information into the secure E-Link environment. Using this information, the security authorities can validate beforehand that the driver and truck are indeed registered to that company. Based on the pre-announcement, customs officers make a risk assessment, whether to issue an inspection or not. The results of the risk assessment are secured and unknown to the company.

Step 3. The truck arrives at the E-Link gate, the driver identifies himself with the ACN identity pass. The E-Link equipment uses biometrics (hand palm recognition) to authenticate the driver. Moreover, license plate recognition is used to authenticate the truck. Subsequently, the truck is weighed. The system verifies whether the driver, truck, company and estimated arrival time match with the pre-announcement. Afterwards, the result of the customs' risk assessment is made known: the driver is shown a display with the loading dock number to go to, or else to go to the customs inspection lane.

Step 4. At a service desk, the driver is authenticated and shows the freight documents. In the future this step can also be removed.

Step 5. At the designated loading dock the goods are unloaded. Driver, truck and freight documentation (bar code or RFID) together identify the freight.

Step 6. After unloading, a representative of the ground handler signs off for delivery of the freight. To do so, he uses his ACN pass to identify himself.

\footnotetext{
${ }^{1}$ http://www.acn.nl/projecten/e-link.aspx , last visited 8th of February 2012.
} 
Step 7. Finally, the truck must again pass the gate, where it is weighed, and the net weight is compared to the expected weight. Before allowing the truck to leave, the system verifies that all possible outstanding issues, e.g., about differences or misunderstandings have been accounted for.

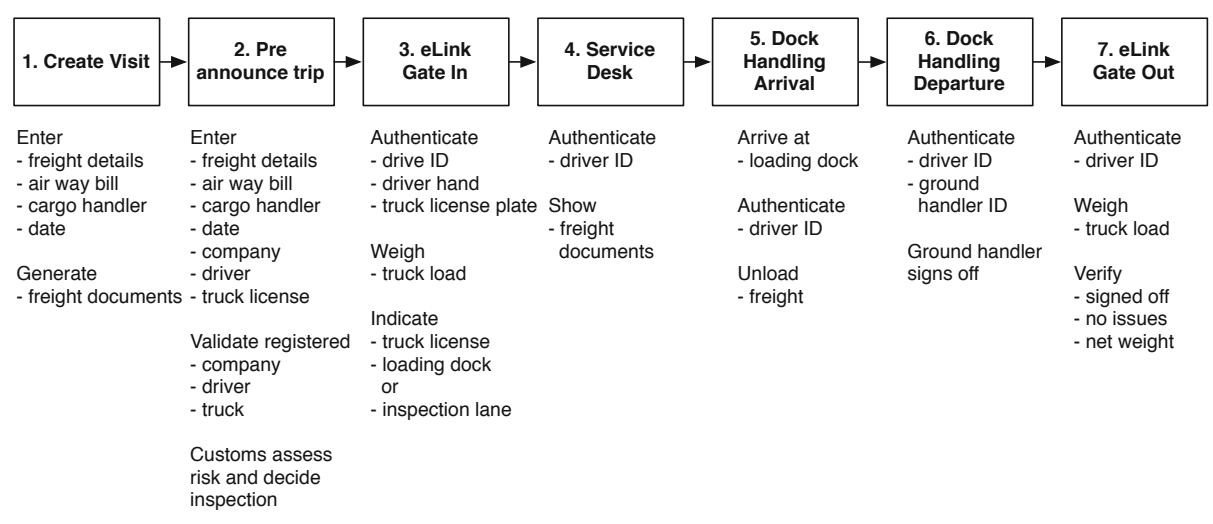

Fig. 3. E-Link process in a nutshell

The case study shows how an electronic procedure can replace the function of a traditional paper process. For example, the ground handler signing off for delivery of the goods remains as before. However, the tools are different. Instead of pink slips, now swipe cards are used in addition to biometrics authentication.

The case study also illustrates our working hypothesis, that the physical flow of goods can be reliably aligned with status updates, represented by trade documents in a shared repository, provided three conditions are met: closed environment, internal consistency and external consistency.

First, E-Link makes use of the trusted trader concept. All participants are members of ACN and must be certified by the customs authorities. Because partners guard their professional reputation at Schiphol Airport, the system of pre-announcements can be trusted. A cargo handler who would abuse the system, would surely be kicked out of the ACN alliance, meaning it is 'out of business'.

Second, The E-Link system uses several kinds of verifications and reconciliations to establish internal consistency. In particular, the system compares data on the commercial trade documents with the pre-announcement. It also compares the listed driver, truck license number and company with a database of companies with registered employees and trucks. It verifies the listed value and amount of goods with common averages for those goods. Moreover, it verifies that the ground handler has signed off for delivery. In this way, the system can establish that either a well-formed delivery transaction was performed, or else, that both parties acknowledge that there are outstanding issues to be resolved.

Third, the system contains several verifications and reconciliations to establish external consistency. In particular, the system is based on a triangulation of 
three data elements: the driver, identified by his ACN pass and authenticated by a biometrics device, the truck, identified and authenticated by license plate number and the freight, identified by the freight documentation (bar code, RFID). As additional circumstantial evidence, the truck is being weighed, both at arrival and departure. The pre-announcement is compared to the actual arrival: expected arrival time, driver, truck and freight must all correspond. If not, the goods are not allowed. At the loading dock, the system again uses identification of a person, the representative of the ground handler, as a proxy for verification of the goods. Given the trusted trader concept this assumption is warranted.

The new process leads to much improved cargo handling. First, it reduces waiting times. Now all arrivals are scheduled in advance, so there will always be enough ground handling capacity. Second, a lengthy customs verification procedure has been removed. Using the pre-announcement, customs can perform their risk assessment off-line and beforehand. In addition, the uncertainty about arrival is reduced, so customs can also schedule their inspections. In addition to these efficiency gains, also the quality of the data has improved. The number of errors and possible exceptions is reduced.

\subsection{Electronic Seals}

In this section we explore the possibility of electronic seals (eSeals) to help establish an integrity relationship between the goods flow and the data flow. eSeals have been studied in the Smart-CM research project [15].

Seals are ancient devices attached to the lock of a container or the lid of a box. To open the container, first the seal must be broken. Although seals can be forged, an unbroken seal counts as physical evidence that a box or container was not opened illicitly. Earlier we discussed the use of a quittance or receipt as evidence that custody over a freight is transferred. Seals can be seen as a physical counterpart to a quittance. Given the countervailing interests of subsequent parties in a supply chain, quittance will only be given, when the party receiving the goods can be reasonably sure that the box contains the goods as stated in the transport documents. When the seal is intact, this provides evidence that the box was not manipulated since it was closed. This ancient function of seals can be greatly enhanced by modern techniques.

eSeals are a particular category of smart seals that are based on active, passive or mixed RFID technology. The internal memory of the RFID chips can save a $\log$ of events. It is possible to assess the correct closure of the seal to avoid fake installations of the seal 18. An eSeal can store in its memory if, by whom and at what time it has been opened and closed correctly. Furthermore, with active RFID technology the eSeal can communicate its state to inspectors. Therefore inspections will depend less on the inspector's skills and experience. Active RFID enables remote and on-the-fly inspection of a box or container in transit. The freight need no longer be stopped. However, as eSeals based on active RFID are battery operated, the consignor must make sure that the on-board battery can keep the eSeal alive until the end of the journey [18]. 
In summary, the following three kinds of security features can be exploited by the utilization of eSeals: (1) the possibility to check for proper closure of an eSeal and to save the operator identity and time of closure in its internal memory, (2) the recording of every (un)authorized operation made on the seal, along with time and date, and (3) the checking of the seal status by remotely gaining access to the seal's memory [18.

We will now review the three components for establishing integrity.

First, the trusted trader concept is used. The World Customs Organization's (WCO) Standards to Secure and Facilitate Global Trade (SAFE) Framework [23] mentions that the integrity of a box or a container brings no value to inspectors if the parties providing the integrity status are not accredited. Companies with the AEO status are considered trustworthy partners for customs authorities in Europe. Companies with the Customs-Trade Partnership Against Terrorism (CTPAT) status are considered trustworthy partners in the USA. Both AEO and C-TPAT certificate holders are audited on compliance with security and other customs relevant standards. In other words, AEO and C-TPAT accreditation gives some assurance to customs that the entry or exit declaration data matches the true content of the container. In addition, through AEO and similarly accredited systems, a bilateral agreement exists between the involved inspection authorities and the accreditation systems are mutually acknowledged [15]. An alternative and stronger way of assuring that a container is closed by a trustworthy party is, for instance, letting it be closed under the local customs authorities' supervision [15. Nevertheless, the identification of the party that has closed and sealed a box or a container remains a key element to establish a proper integrity relationship between the goods and the data.

Second, the tracking and tracing facilities of eSeals make it possible to establish a virtual secure pipeline, within the otherwise untrusted general supply chain. For example, in a pilot study, the beer brewer Heineken has demonstrated that it can be fully 'in control' of the export of premium beers by using electronic seals [21. In general, to utilize eSeals, the security status information from the eSeals must be uniquely linked to cargo data. Once established, an integrity relationship between goods and data remains intact as long as the parties that close, seal and open a container are either trusted or are public inspection authorities. As it is possible to save the identity of the operator who has opened or closed an eSeal, relationships can be formed between operators closing the seals and operators opening the seals. In particular, an eSeal that is attached to a package (a box) packed inside another sealed package (a container), can only be opened after the seal on the outer container is opened. This also means that operators who are authorized to open eSeals on boxes that are aggregated in a container, should in principle also be authorized to open (and re-seal) that outer container. This implies that the order in which seals of aggregated packages are opened, should be the reverse of the order in which the seals of those packages are closed.

Third, establishing external consistency is the whole point of having seals. The crucial event is the closing or opening of a package. Therefore, there are strict procedures about opening or closing a seal. Because the procedures of parties 
are being audited, as part of the AEO or C-TPAT accreditation schemes, we can be reasonably certain that those procedures are being observed.

\section{Discussion}

In the paper we have described theoretical approaches to make sure that trade documents in a virtual data pipeline correspond to reality. In practice, adopting and implementing such measures is complex. The development is likely to meet a number of legal, commercial, political and technical challenges. Therefore, we will now briefly discuss two challenges and possible ways to overcome them.

Challenge 1. Disrupting the process. Most of the control measures we have described are not part of the primary process as they are added. However, control measures should not interfere too much with the continuation of the supply chain, otherwise they are circumvented. The flow should not be disrupted.

Consider the following example. At a loading dock in a harbor, cranes are equipped with a weighing device, which automatically weighs the container. The following control is implemented: when the weight exceeds the maximum load, the crane will stop working. The idea is that too heavy containers will affect the safety of the loading process and the ship. However, in the experience of customs officers, the control is generally switched off. A delay is simply too costly.

Suppose the controls were set up in a different way, where the crane registers the weight of each container and sums the total weigh of a freight. Now, both cargo handler and shipping company have an independent number to verify the accuracy of the trade documentation. It can also be used by customs' officers: excess weight requires some explanation. However, importantly, such differences can be settled later and will not disrupt the loading process.

Challenge 2. Information quality and role in the chain. Customs have the duty to stop undesired goods like drugs, weapons, or counterfeit goods at the border (stop function). The customs office would like to use the virtual data pipeline for targeting its inspections. Nowadays, customs use a risk-based inspection approach. Based on stated properties of the goods, the shipper, the country of origin and destination, etc., certain freights are selected for inspection. However, this requires that the data are accurate and complete.

However, currently the data in customs declarations is of low quality. The data are incomplete, late, or no longer accurate after re-routing. Customs declarations are filled in by the carrier. However, the carrier is usually not in a position to provide more accurate and detailed information; such information is not needed for fulfilling his role in the supply chain. What is more, telling the carrier about the contents of boxes would increase the likelihood of theft. Participants will be very reluctant to share such information.

Therefore, supply chain visibility for regulatory purposes can only be a success, when the legal responsibility to fill out the details of the goods is somehow transferred to the supply chain as a whole, involving the consigner and the consignee. This may be very difficult to achieve from an administrative point of 
view. Moreover, to make the concept of a secure pipeline acceptable for commercial parties, it should be possible to demonstrate that parties will only get access to shared information in the pipeline, which is needed for their task in the chain (principle of least privilege).

\section{Conclusions and Future Research}

In this paper we have looked at supply chain visibility: reusing commercially available data to trace goods along the supply chain and make them visible for regulatory purposes. A particular approach is the virtual data pipeline concept: an infrastructure for exchanging trade documents among trusted traders [14]. For successful adoption, trust in the reliability of the data in the virtual pipeline is crucial. In this paper, we have looked at theories about the way in which the trade documents which are being being exchanged in the virtual data pipeline can be undeniably linked to the physical flow of goods.

We argued that in order to do so, we need three kinds of control measures: (i) All participating companies are known and certified (trusted traders). This warrants the assumption that participants' employees can generally be trusted to do their work, for instance, to apply seals or sign off according to procedures. (ii) Measures to establish internal validity. Database management systems can maintain integrity constraints for the data being stored. The integrity constraints are derived from the meaning of the documents in the trade process. All steps in the process are interpreted as status updates concerning the exchange of value, of goods, or of information. These steps in the process should together form a well-formed transaction, from an economic point of view. The system can verify specific reconciliation relations, derived on the basis of accounting theory.

(iii) Measures to establish external validity. All control measures are rendered powerless, unless there are basic physical security measures in place, like fences, doors, locks and keys. These measures protect the integrity of a storage space, such as a warehouse, truck or container. In addition, there are modern technological control measures, like authentication devices (biometrics) or electronic seals (RFID), which have taken over the function of their ancient and proven predecessors, but have advanced them. In particular, these new technologies make it possible to trace the goods or the people handling them from a distance.

The control measures are linked in a three layered architecture, consisting of the business process or value layer, the information or data layer and the physical infrastructure layer. The relationship between layers is a representational one: events or facts at the physical layer generate evidence which counts as or constitutes evidence of institutional events or facts at the business layer.

The approach is illustrated by two cases. The E-Link project about air cargo export at Schiphol Airport illustrates that it makes sense to share information early on, and thereby reduce waiting time and uncertainty. The electronic seals developed in the Smart-CM project show that much security related supply chain information can be stored locally in the seals themselves which makes the chain transparent. In future research we will investigate this line of reasoning further 
and show how to model and formalize a specific set of continuity equations for each of the layers, and align them, for a specific non-trivial case.

Acknowledgments. We are grateful to our colleague Yao-Hua Tan for valuable input. We would like to thank Aike Müller for information about the E-Link project at Schiphol airport.

\section{References}

1. Aldewereld, H.: Autonomy vs. Conformity - An Institutional Perspective on Norms and Protocols. Ph.D. thesis. Universiteit Utrecht (2007)

2. Blokdijk, J., Drieënhuizen, F., Wallage, P.: Reflections on Auditing Theory, A contribution from the Netherlands. Limperg Institute Amsterdam (1995)

3. Boritz, J.: Is practitioners' views on core concepts of information integrity. International Journal of Accounting Information Systems 6(4), 260-279 (2005)

4. Chen, S., Drake, J., Tsai, W.: Database requirements for a software engineering environment: criteria and empirical evaluation. Information and Software Technology 35(3), 149-161 (1993)

5. Clark, D., Wilson, D.: A comparison of commercial and military computer security policies. In: IEEE Symposium on Security and Privacy, pp. 184-194 (1987)

6. European Commission: AEO guidelines. Tech. Rep. TAXUD/2006/1450, Directorate-General Taxation and Customs Union (2007)

7. Dietz, J.L.: The deep structure of business processes. Communications of the ACM 49(5), 59-64 (2006)

8. FIPS: Minimum security requirements for federal information and information systems. Tech. rep., U.S. National Institute of Standards in Technology, NIST (2006)

9. Ghaeb, J., Chebil, J.: An oblique-matrix technique for data integrity assurance. Computers \& Security 28(1-2), 94-99 (2009)

10. Governatori, G., Sadiq, S.: The journey to business process compliance, pp. 426 445. IGI Global (2009)

11. Grefen, P., Apers, P.: Integrity control in relational database systems - an overview. Data and Knowledge Engineering 10(2), 187-223 (1993)

12. Grossi, D.: Designing Invisible Handcuffs: Formal Investigations in Institutions and Organizations for Multi-agent Systems. Ph.D. thesis. Universiteit Utrecht (2007)

13. Hesketh, D.: Weaknesses in the supply chain: who packed the box? World Customs Journal 4(2), 3-20 (2010)

14. Hofman, W.: Supply chain risk analysis with linked open data. In: Vermaas, P.E., Dignum, V. (eds.) FOMI 2011, pp. 77-87. IOS Press, Amsterdam (2011)

15. INTEGRITY, SMART-CM: Global container supply chain compendium (2008)

16. Klein, R., Rai, A.: Interfirm strategic information flows in logistics supply chain relationships. MIS Quarterly 33(4), 735-762 (2009)

17. Müller, A.: Re-engineering Security: A risk based approach for information security focused business process re-design. Master's thesis. Erasmus Universiteit (2012)

18. Rizzo, F., Barboni, M., Faggion, L., Azzalin, G., Sironi, M.: Improved security for commercial container transports using an innovative active RFID system. Journal of Network and Computer Applications 34(3), 846-852 (2011)

19. Searle, J.R.: The Construction of Social Reality. The Free Press (1995)

20. Starreveld, R.W., de Mare, B., Joels, E.: Bestuurlijke Informatieverzorging, vol. 1. Samsom, Alphen aan den Rijn (1994) (in Dutch) 
21. Tan, Y., Bjørn-Andersen, N., Klein, S., Rukanova, B. (eds.): Accelerating Global Supply Chains with IT-Innovation. Springer, Berlin (2011)

22. Vasarhelyi, M.A., Alles, M., Kogan, A.: Principles of analytic monitoring for continuous assurance. J. of Emerging Technologies in Accounting 1(1), 1-21 (2004)

23. WCO: SAFE framework of standards to secure and facilitate global trade (2011)

24. Weigand, H., de Moor, A.: Workflow analysis with communication norms. Data and Knowledge Engineering 47(3), 349-369 (2003)

25. Yu, L.: Linked open data. In: A Developer's Guide to the Semantic Web, pp. 409466. Springer, Heidelberg (2011) 